\title{
Hydraulic Domestic Heating by Throttling
}

\author{
Mohammad A. K. Alia, Tariq Younes, Hussein Sarhan \\ Al-Balqa Applied University, Faculty of Engineering Technolog, Al-Salt, Jordan \\ E-mail:makalalia2000@yahoo.com, \{tariqmog, sarhan_52\}@hotmail.com \\ Received February 3, 2010; revised March 15, 2010; accepted March 25, 2010
}

\begin{abstract}
In this work an experimental investigation was carried out in order to explore the possibility of realizing a domestic heating system by throttling hydraulic oil. Considering the continuous increasing price of diesel oil, this work gains unique importance. Generating heat directly by throttling is realized using a simple environment friendly system which does not require oil transportation and storage, and eliminates the need for chimneys and annual preventive maintenance, as it is the case with heating by utilizing oil burners, which is prevailing in Jordan. Experimental results show that it is possible to raise the room temperature up to $70^{\circ} \mathrm{C}$ during 15 minutes which is not a limit value. Experimental results show that temperature rate could be increased by selecting the appropriate pump power and by connecting a number of throttles in parallel.
\end{abstract}

Keywords: Throttling, Pressure Drop, Hydraulic Oil, Heating

\section{Introduction}

It is an established fact that heat is generated in a fixed displacement hydraulic system whenever fluid is throttled from high pressure to low pressure without doing any work. This generated heat is normally taken as a measure of system efficiency. Although many thermo and fluid references [1,2], describe the generated heat as "losses", fluid flow and pressure drop may be converted to work.

Nowadays, this phenomenon finds a practical application in swimming pools in order to increase heating system efficiency. For this particular case water circulates through a hydraulic motor/turbine, via a heat exchanger, where water is heated. Further it is directed through nozzles in order to be heated for the second time by admixing it, by means of its turbulence with the pool water. Another application is the adjustment of the temperature of large area heating system by automatic adjustment of the throttle degree, so that the temperature difference between the feed-line and the return line of the heating loop stays the same.

It is a common practice in our daily life to directly convert electrical energy into heat energy by using electric wire coils. In such a case the wasted electrical power remains a target. What we are suggesting is to make an analogue process by converting hydraulic energy into heat energy by utilizing a hydraulic resistance (throttle). Considering the continuous increasing price of oil, it becomes apparent that direct consumption of electrical energy by throttling will be more economical then generating heat energy using separate individual domestic oil burners, which is the prevailing heating technology in Jordan. There will be no need for oil storage and oil transportation. Add to this the suggested heating system does not require chimneys and does not involve any contamination. Moreover the system is very simple and less complicated when compared with diesel burner equipment. Building on the above, the target of this work is to experimentally investigate the possibility of realizing a hydraulic domestic heating system by throttling.

\section{Why so Much Heat is Generated during Throttling?}

Heating by throttling is generated by friction due to pressurized oil forced into restriction [3]. The higher friction will be between the oil and the inner surfaces of the throttling valve. By circulating the heated oil during a specific interval of time a considerable temperature rise will take place, and as a result of that surrounding environment will be heated. Fluids in motion are subjected by various resistances, which are due to friction. Friction may occur between the fluid and the pipe work and within the fluid as sliding between adjacent layers of the fluid takes place, which is normally characteristic for turbulent flow. 
Although friction is an important factor, friction alone does not seem to account for the heat generation in the hydraulic throttling process. A reasonable explanation is to refer to the long molecular chain which gives oil its viscosity and lubricity. As the fluid stream passes though the pipes, molecules at the edge of the stream tend to adhere to the inner walls of pipes. This slows down the outer surface of the flow, and causes stresses and tearing of molecular chains. Heat is then generated when the molecular bonds are broken and re-formed. This is the reason for the addition of heat to any liquid in motion. The lower the velocity of the liquid is, the smaller the ratio of stressed molecular chains, as well as the amount of heat build-up in the liquid. Now it is clear why turbulent motion, which is associated with relatively high speed, is accompanied with heat build-up. However, in a throttling situation, energy from the prime mover is transferred into liquid by the pump. Molecules of the liquid get compressed and in turn it slows the molecular activity. When we throttle a system, we allow some of the compressed liquid to escape to the low pressure side, where the liquid decompresses and increases at the same time its molecular activity and releases energy into heat. The only energy that oil can store is potential energy. If it cannot release its energy by transferring it to kinetic energy, sound, and force, it will always release it in the form of heat.

\section{Which is the Preferred Heating Medium?}

Nowadays water is the dominating heating medium in Jordan. This is because it is cheap. As water causes rust and corrosion it must be chemically treated and heating network must be flushed annually. The specific heat of water is $4186 \mathrm{~J} / \mathrm{Kg} . \mathrm{C}^{\circ}$ [4]. Concerning mineral oil, its specific heat is $1966 \mathrm{~J} / \mathrm{Kg} . \mathrm{C}^{\circ}$ and the heating network is not subjected to rust or corrosion. This means that time interval required to raise the room temperature to the preset value is two times shorter than that required when using water. Considering the working temperature range for water, practically, it is limited to $90^{\circ} \mathrm{C}$ in order to avoid water evaporation. For oil it is limited to $82^{\circ} \mathrm{C}$ in order to keep oil viscosity not deteriorated [4]. So practically both water and oil have the same temperature working range. When applying hydraulic throttling, cavitation of the throttling valve becomes an important issue. In water hydraulic valve, cavitation is more likely to happen than in oil hydraulic one. Water has a low viscosity and high vapor pressure. Its vapor pressure increases rapidly when temperature rises [5]. Because of high vapor pressure of water cavitation is more likely to happen especially when the pressure drop across the valve orifice is large. As a result of higher density of water than oil, the pressure impact resulting from cavitation in water hydraulic system is more serious than in an oil system and the flow coefficient of the throttle using water as a pressure medium is larger than that using oil as a working medium. Considering the above, oil is recommended as the pressure medium.

\section{Experiment Setup and Hydraulic Circuit Block Diagram}

The experiment setup and hydraulic circuit block diagram are shown in Figures 1(a) and 1(b).

The circuit includes a gear pump, pressure relief valve, pressure gauge, throttle valve and a tank. In addition to that a manifold piping network may be added to the circuit in order to allow throttling through one valve only, two throttling valves in series or in parallel, and three throttling valves in series or in parallel. This is shown in Figure 2.

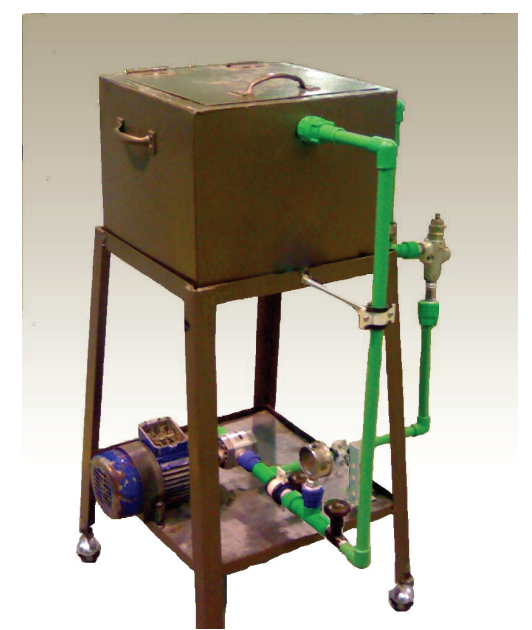

(a)

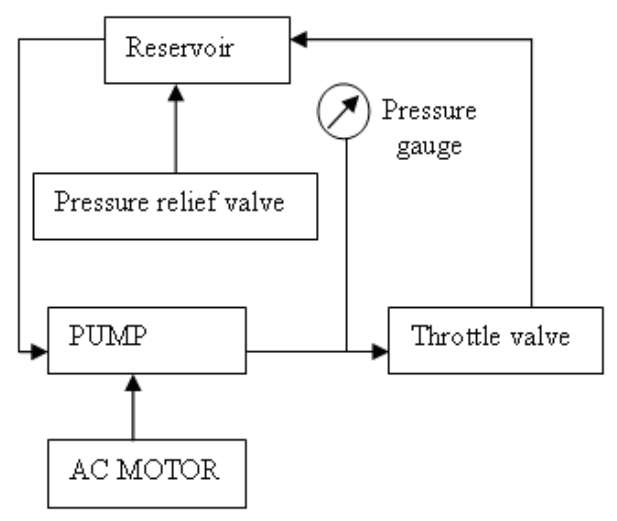

(b)

Figure 1. (a) Experiment setup; (b) Hydraulic circuit block diagram. 
The tank is insulated by fiber glass and includes an internal oil distributor. The tank dimensions are $50 \times 50 \times$ $25 \mathrm{~cm}$. Oil volume was 15 liters. The tank represents the oil filled radiators in real domestic heating system. Circulation pump is not shown in the experimental setup because the distance between the output of the throttle valve and the tank is short.

Temperature is measured every three minutes using a glass thermometer. The pressure relief valve is installed for safety to prevent pressurization and possible failure.

Throttle valve is a manually operated multiturn valve. A $3 \mathrm{~kW}$ Power gear pump is selected in order to realize high pressure. The rigid design of gears and housing of the pump allow for high pressures and the ability to pump highly viscous liquids. Gear pumps are ideally suitable for working pressure below 120 bars. It deserves to note that gear pumps have high power dissipation and deliver a continuous supply with no pulsations, which aids the heating process and gives a uniform oil flow.

\section{Experimental Procedure and Experimental Results}

The first experiment was carried out using one throttle valve. In order to adjust the throttle opening it was closed completely, and then it was opened manually by rotating its wheel 2, 3, 4, 5 turns successively. The pump was started manually and oil temperature was taken every three minutes until oil temperature has reached a specific value. Room temperature was $21^{\circ} \mathrm{C}$. After taking the readings of the specific number of turns the system was left to cool to room temperature. Experiment results are given in Table 1 and Figure 3.

The second experiment was carried out using three identical throttles with the same opening ( 5 turns). Table

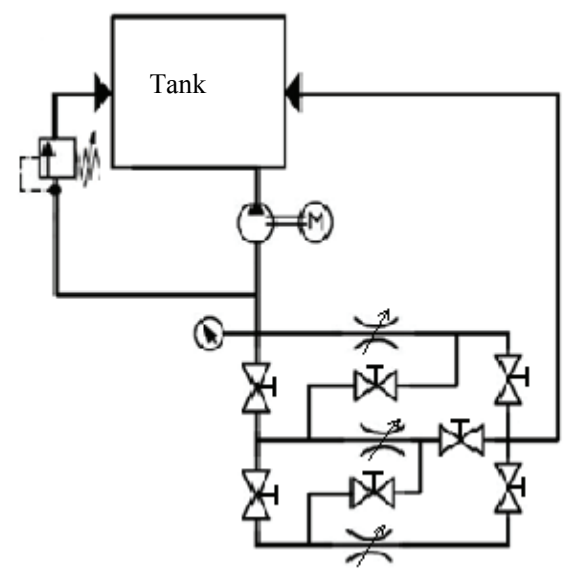

Figure 2. Manifold piping network connected with the hydraulic circuit.
2 and Figure 4 show the experimental results when only one throttle was used, then two throttles were used in parallel connection, and at the end three throttles were used in parallel connection.

Normally a real domestic heating system is controlled automatically, using an ON-OFF controller with an acceptable hysteresis loop width and an appropriate temperature transducer [6]. For a finer control a proportional

Table 1. Experimental results using single throttle with different settings.

\begin{tabular}{|c|c|c|}
\hline \multirow{7}{*}{2 turns } & Time[min] & $\operatorname{Temp}\left[{ }^{\circ} \mathrm{C}\right]$ \\
\hline & 0 & 21 \\
\hline & 3 & 36 \\
\hline & 6 & 49 \\
\hline & 9 & 57 \\
\hline & 12 & 65 \\
\hline & 15 & 70 \\
\hline \multirow{6}{*}{3 turns } & 0 & 21 \\
\hline & 3 & 34 \\
\hline & 6 & 47 \\
\hline & 9 & 55 \\
\hline & 12 & 63 \\
\hline & 15 & 67 \\
\hline \multirow{6}{*}{4 turns } & 0 & 21 \\
\hline & 3 & 32 \\
\hline & 6 & 42 \\
\hline & 9 & 51 \\
\hline & 12 & 59 \\
\hline & 15 & 62 \\
\hline \multirow{6}{*}{5 turns } & 0 & 21 \\
\hline & 3 & 30 \\
\hline & 6 & 41 \\
\hline & 9 & 49 \\
\hline & 12 & 55 \\
\hline & 15 & 60 \\
\hline
\end{tabular}

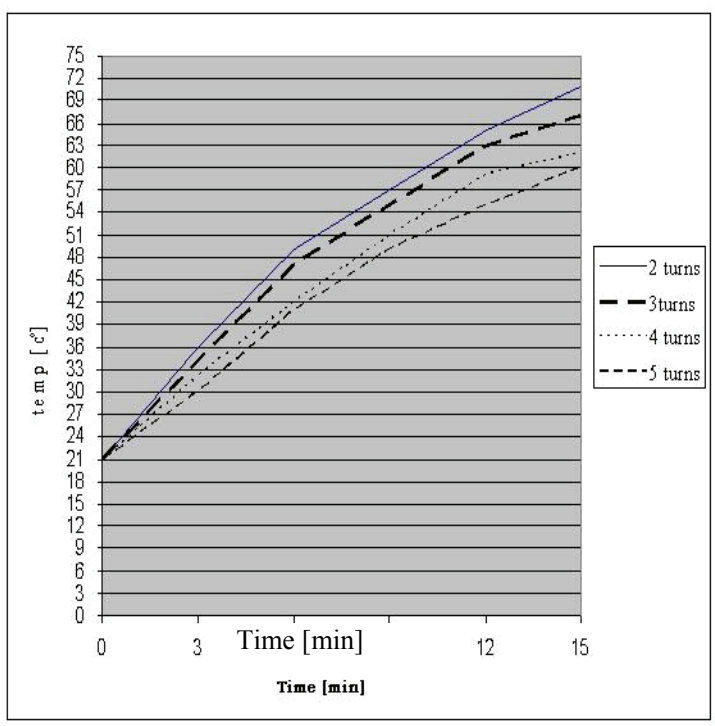

Figure 3. Temperature versus time graph at single throttle. 
Table 2. Experimental results using three parallel throttle of the same settings.

\begin{tabular}{lll}
\hline & Time $[\mathrm{min}]$ & $\mathrm{Temp}\left[{ }^{\circ} \mathrm{C}\right]$ \\
& 0 & 21 \\
1 Throttle $(5 \mathrm{t})$ & 3 & 30 \\
& 6 & 41 \\
& 9 & 49 \\
& 12 & 55 \\
& 15 & 60 \\
& 0 & 21 \\
& 3 & 33 \\
& 6 & 44 \\
& 9 & 53 \\
& 12 & 62 \\
& 15 & 67 \\
& 0 & 21 \\
& 3 & 37 \\
& 6 & 46 \\
Throttles $(5 \mathrm{t})$ & 9 & 54 \\
& 12 & 63 \\
& 15 & 68 \\
\hline
\end{tabular}

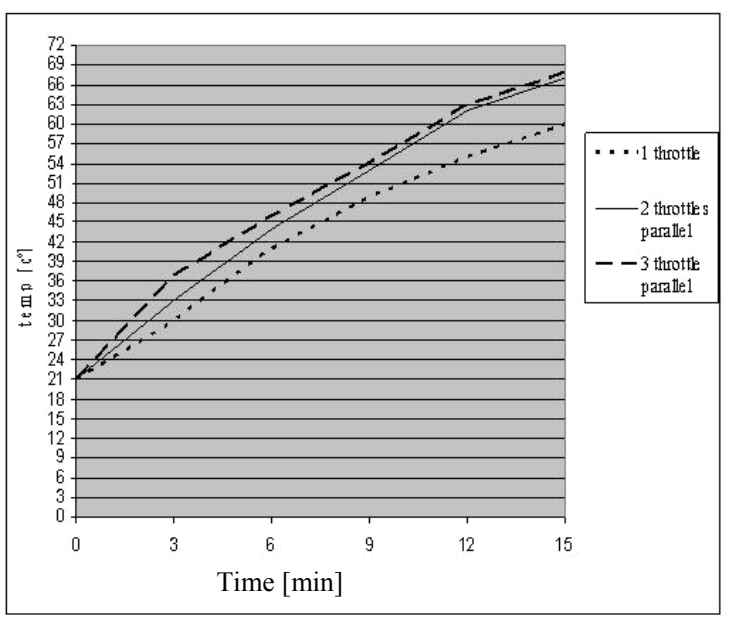

Figure 4. Temperature versus time graph at one, two and three throttles in parallel.

throttle valve may be utilized in order to control the valve internal plug position as a function of system temperature.

\section{Design and Practical Considerations}

As a rule of thumb, total losses are estimated by $25 \%$ of the input power of the most common hydraulic systems [7]. For a heating by throttling system there is no work done. The whole input power has to be converted into heat power. This heating system is in essence on oil/air heat exchanger. Such an exchanger may be rated ac- cording to the amount of heat dissipated for each degree of difference between the maximum oil temperature and the ambient temperature at a given flow of oil through the throttle. The heat load of the hydraulic system, which corresponds to pump power, can be determined by measuring the radiators temperature at start up and again after the heating system has been in operation for a measured time interval by using the formula (Cooling and Heating, 2006),

$$
P=\frac{V \times \Delta T}{32.4 \times \Delta t}
$$

where

$P \quad$-Power (heat load) in $\mathrm{kW}$

$V \quad$-tank volume in liters

$\Delta T \quad$-temperature change $\left(T_{2}-T_{1}\right)$ in ${ }^{\circ} \mathrm{C}$

$\Delta t \quad$ - time change $\left(t_{2}-t_{1}\right)$ in minutes

If $\Delta T$ is equal to $(75-15=60)^{\circ} \mathrm{C}$, which is a real practical value

$\Delta t \quad$ is equal to 15 minutes

then,

$$
P=\frac{V \times 60}{32.5 \times 15}=0.123 \mathrm{~V} \quad \mathrm{~kW}
$$

If the temperature controller works in an OFF-ON mode with a hysteresis bandwidth equal to $6^{\circ} \mathrm{C}$ and it is required that the controller must clear the temperature deviation within (3) minutes, then the required power for each working cycle shall be equal to:

$$
P=\frac{V \times 3}{32.5 \times 3}=0.03 V \mathrm{~kW}
$$

This relationship may be utilized in order to estimate the consumed power for any time period.

\section{Conclusions}

- Experimental results show that it is practically possible to make use of the throttling effect in hydraulic systems in order to realize commercial industrial domestic heating systems.

- Oil is the preferred pressure medium. It has a low specific heat capacity, it does not cause rust or corrosion and its working temperature range is practically the same as that of water. In addition to that valve cavitation is severely eliminated.

- Temperature rise-time may be controlled utilizing an adjustable or controllable one throttle or more than one throttle connected in parallel.

- Given a simple method for heating load evaluation and for the determination of the consumed power.

\section{References}

[1] Y. A. Cengel and M. A. Boles, "Thermodynamics an Engineering Approach,” McGraw-Hill, USA, 2006. 
[2] Hydraulic Supermarket, "Cooling and Heating," 2006. http:// www.industrialhydrauliccontrol.com

[3] Hydraulic Secrets Revealed, http://www.insidersecretstohydraulic.com

[4] P. Frank, "Fundamentals of Heat and Mass Transfer," John Wiley and Sons, UK, 2004.
[5] J. Michael, "Industrial Control Electronics Applications and Design," Prentice-Hall International Inc., USA, 1990.

[6] M. Prasad, "Refrigeration and Airconditioning Data Book," New Age International, India, 2009.

[7] R. L. Mott, "Applied Fluid Mechanics," Prentice Hall, USA, 2009. 